\title{
Vaccine-related serious adverse events might have been under-recognized in the pivotal HPV vaccine randomized trial
}

\author{
Manuel Martínez-Lavín ${ }^{1}$ (iD
}

Received: 26 January 2017 / Accepted: 7 February 2017 / Published online: 16 February 2017

(C) International League of Associations for Rheumatology (ILAR) 2017

To the Editor:

After seeing several healthy girls developing a severe chronic illness soon after HPV vaccination [1], I analyzed all HPV vaccine randomized controlled trials.

The overwhelming majority of pre-licensure HPV vaccine randomized trials did not use inert placebo as comparator. The largest nine-valent HPV immunization trial [2] compared the newly developed nine-valent HPV vaccine vs. the four-valent HPV formulation. The innovative nine-valent HPV dose has more than double HPV viruslike particles and aluminum adjuvant than the previous formulation. Double-blind safety analysis contrasted 7071 subjects immunized with the nine-valent vaccine vs. 7078 who had the four-valent dose. The nine-valent cohort had significantly more systemic serious adverse events; $n=233(3.3 \%)$ vs. $n=183(2.6 \%)$ in the other group. Our calculated $2 \times 2$ contingency table $p$ value was 0.0125 . Oddly, only two subjects $(0 \%)$ in each group were judged to have a vaccine-related serious adverse event. The authors did not comment on this incongruity.
This discrepancy arising from a pivotal large randomized double-blind trial suggests that nine-valent HPV vaccine-related serious adverse events were under-recognized. This emerging information casts further doubt on HPV vaccine safety.

Compliance with ethical standards

Disclosures None.

\section{References}

1. Martínez-Lavín M, Martínez-Martínez LA, Reyes-Loyola P (2015) HPV vaccination syndrome. A questionnaire-based study. Clin Rheumatol 34:1981-1983

2. Joura EA, Giuliano AR, Iversen OE et al (2015) A 9-valent HPV vaccine against infection and intraepithelial neoplasia in women. $\mathrm{N}$ Engl J Med 372:711-723
Manuel Martínez-Lavín

drmartinezlavin@gmail.com

1 Rheumatology Department, National Institute of Cardiology, Mexico City, Mexico 